\title{
RESHAPING TECHNICAL DIFFICULTY IN RELATION TO CHANGES IN THE CODE OF POINTS
}

\author{
Ştefania CHIRIAC ${ }^{*}$ \\ ${ }^{1}$ National University of Physical Education and Sport, Faculty of Physical Education and Sport, \\ Bucharest, Romania \\ *Corresponding author: stefania.chiriac24@gmail.com
}

https://doi.org/10.35189/dpeskj.2020.59.s.11

\begin{abstract}
The current paper aims to highlight changes in the Code of Points over the last 15 years from the perspective of the Difficulty criterion, with an impact on the development trends in rhythmic gymnastics worldwide. The outcome of this study relates to finding significant quantitative and qualitative cues in order to prepare subsequent training and competition models for the Romanian gymnasts. The main research methods used were: literature review, video analysis and observation, as well as graphical representation. The successive editions of the FIG Code of Points have undergone significant changes since 2013, creating a balance between body difficulty and apparatus difficulty. The present Code of Points places emphasis on the Difficulty criterion, for which the 10-point limit has been eliminated. The apparatus difficulty elements have also been redefined and the values of deductions for technical faults have been substantially increased. This set of provisions impacts the way in which body difficulty, dance steps combination, apparatus difficulty and dynamic elements with rotation are inserted in the routines from both a numerical and value standpoint. As a conclusion, in order to build a strategy to improve the performance of Romanian gymnasts in international competitions, a careful analysis of the exercises performed in large-scale competitions is required. The data extracted and interpreted can represent an important resource of information through which technicians can analyse the new trends in this sport and adapt the provisions of the FIG Code of Points so that the potential of each gymnast can be maximally exploited.
\end{abstract}

Keywords: rhythmic gymnastics, Code of Points, technical difficulty.

\section{Introduction}

In the 21st century, world sport has developed at an accelerated pace, aiming to achieve record performances in all specific branches and events. This unprecedented development attracts more and more sport consumers who appreciate the spectacular technical performance and the excitement before finding out the winner, given that today's athletes are of extremely close values.

This developmental process also includes rhythmic gymnastics, a highly complex sport that combines the attributes of the technique with the aspects of body aesthetics and expressivity, which are unique in the context of sport branches.

In the current sphere of high-performance sports, rhythmic gymnastics is going through a transition period, facing major changes in the last decade, which are translated into the rules imposed by each edition of the FIG Code of Points (CoP).

This sport has undergone impressive changes in recent decades, primarily due to the increased level of technical skills resulting from the constant revision of the requirements imposed by the FIG Code of Points. These new requirements highlight the importance of performing elements with a high degree of difficulty, which involves the development of appropriate physical support to enhance the motor performance of gymnasts. 


\section{Current knowledge in the review of literature}

In an attempt to turn gymnastics competitions into highly attractive events for the general public, the International Gymnastics Federation (FIG) seeks to regulate this issue using an instrument specific to all branches of Olympic gymnastics, namely the Difficulty criterion. In 2017, FIG decides to eliminate the upper limit for this criterion and consequently substantial changes have been noted throughout the years in both the related scores and the final scores.

As an example, Figure 1 shows the spectacular evolution of the total score achieved by gymnast D.A. (RUS) at the last three editions of the World Senior Championships in the individual all-around event (the scores result from the cumulation of the 4 marks obtained for the hoop, ball, clubs and ribbon exercises).

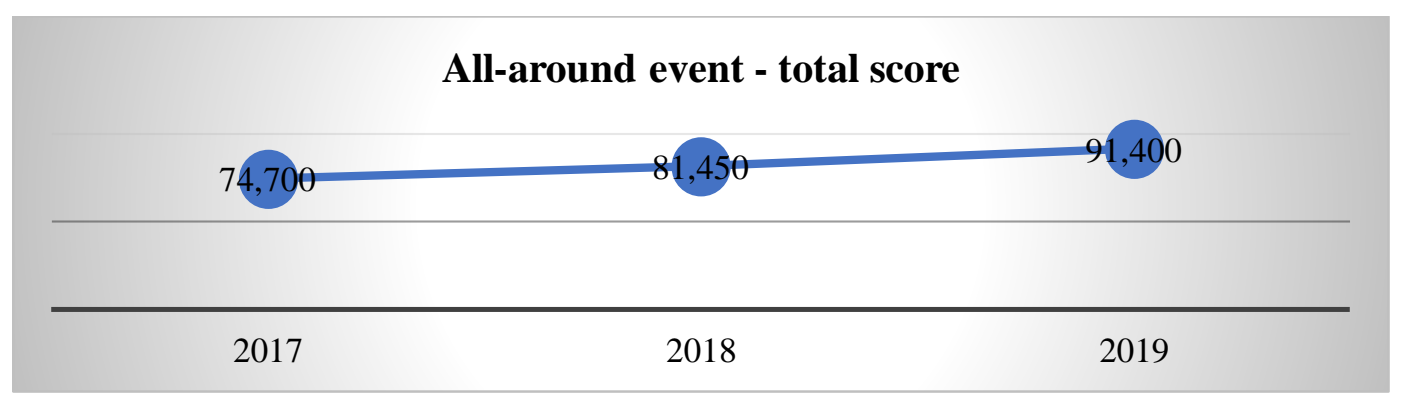

Figure 1. Evolution of the total score in the all-around event for the World Champion in the senior category during the period 2017-2019

The opening of the Difficulty score in rhythmic gymnastics compelled coaches to search for solutions to increase the total value of this evaluation criterion, which is divided into two components, namely the technique of body movements and apparatus technical elements. Figure 2 illustrates the weight in the final score of the components related to the Difficulty criterion and the artistic component, as part of the Execution criterion.

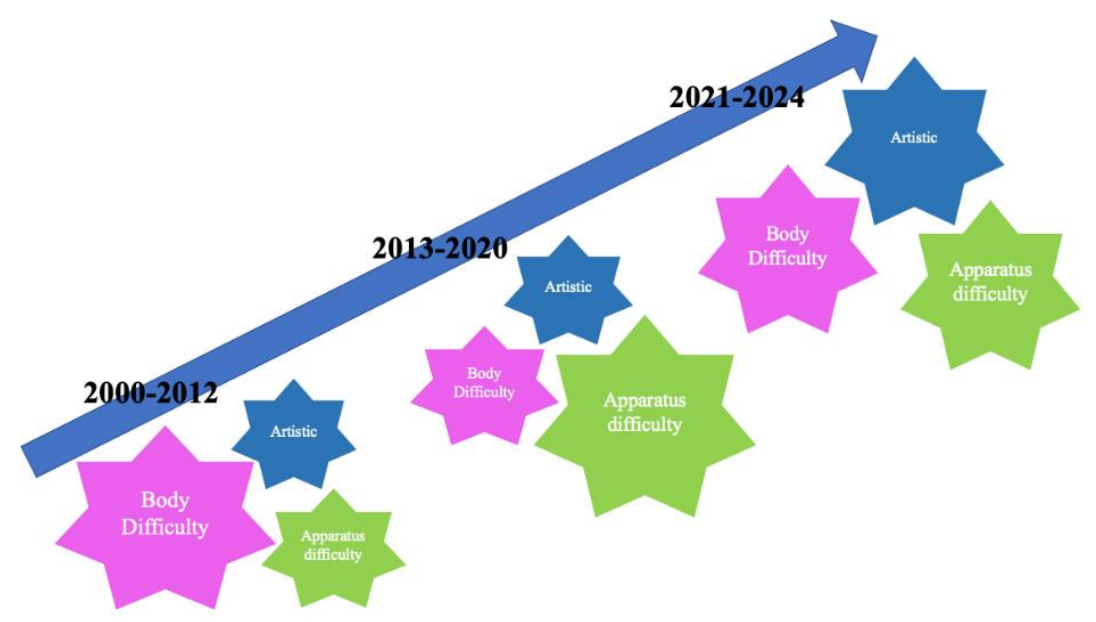

Figure 2. Evolution of the components specific to rhythmic gymnastics and proposals for the 2021-2024 Olympic cycle 
Nowadays, the International Gymnastics Federation needs to conduct a critical analysis of the competitions and performance of gymnasts in this Olympic cycle to see if the regulations implemented in the last 3 years have been beneficial to elite rhythmic gymnastics.

A 20-year longitudinal study of individual routines in elite rhythmic gymnastics (SierraPalmeiro et al., 2019) emphasised changes in the Code of Points and their effects on increasing difficulty and technical mastery, as well as diversity in apparatus handling. The evolution of the number of technical elements and the total score over a two-decade period revealed a general trend of raising both the spectacular impact and the complexity of the compositions (Figures 3 and 4, where vertical lines indicate changes in the Code of Points). Each new Code requires limitless creativity from coaches so that the gymnast-apparatus interaction provides varied and unpredictable movement patterns.

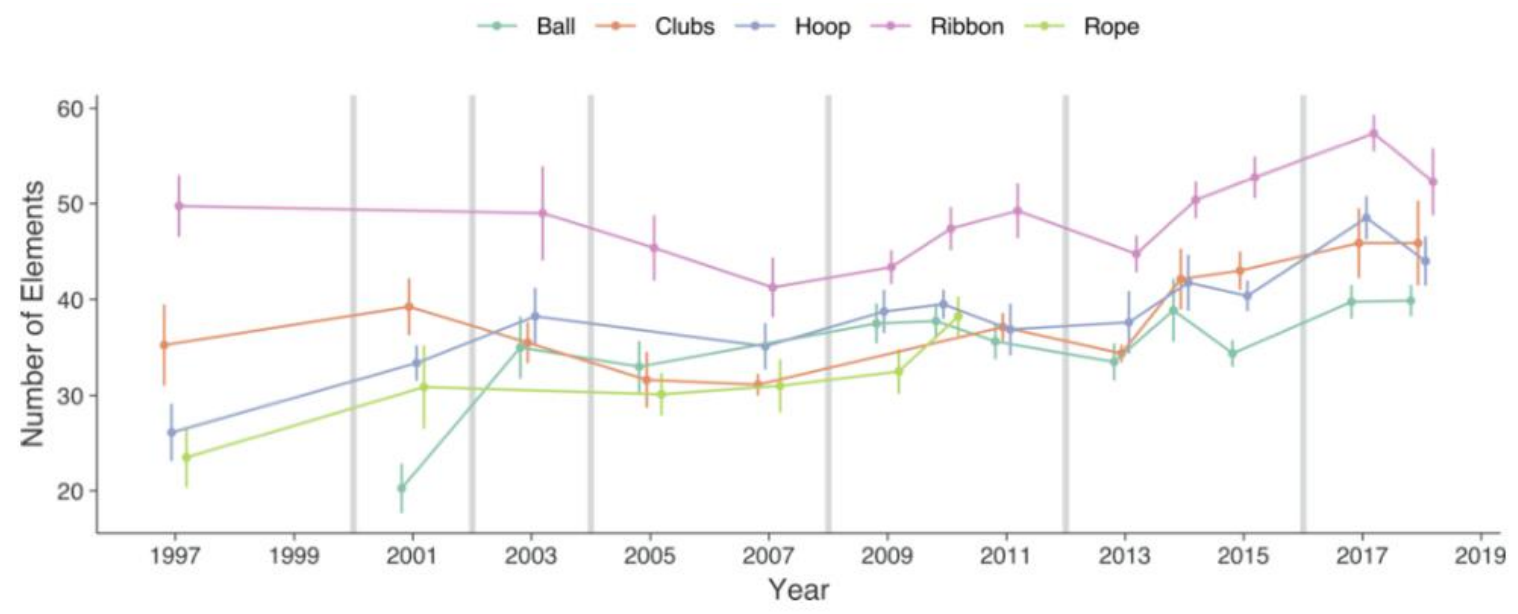

Figure 3. Number of difficulty elements over time for each apparatus

(Sierra-Palmeiro et al., 2019)

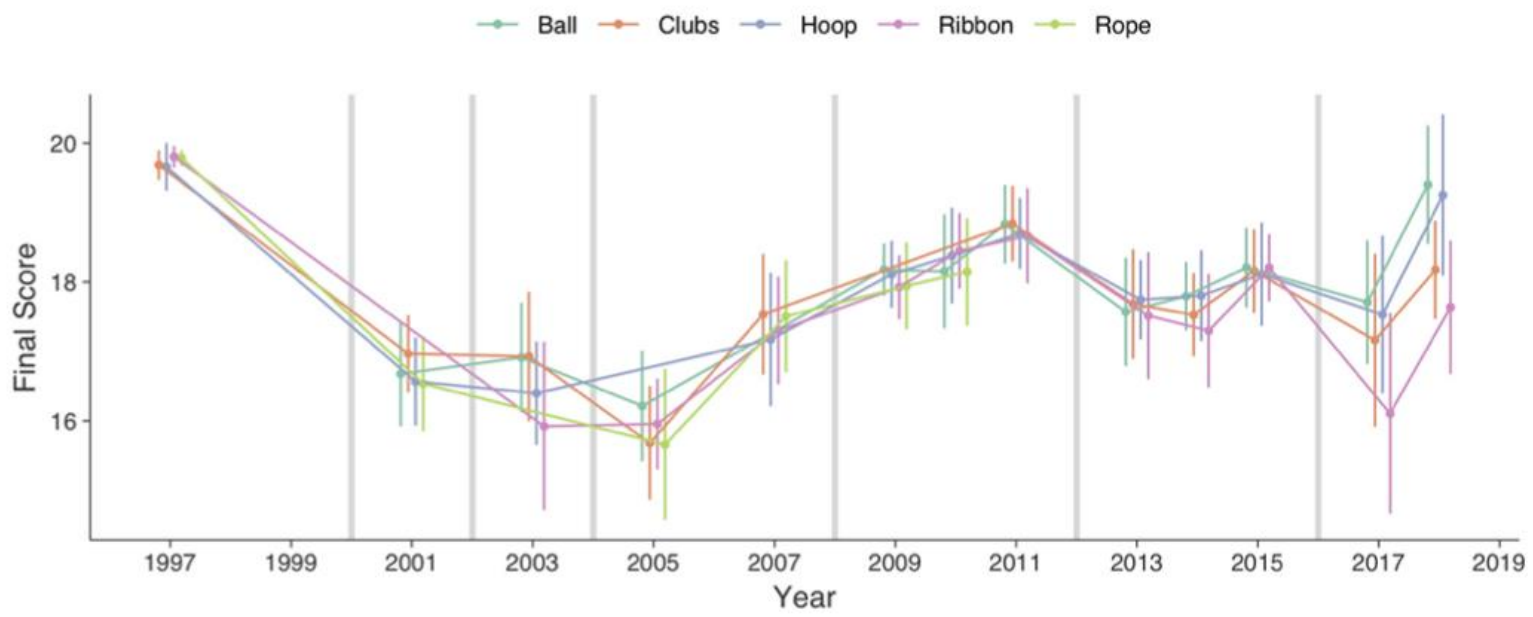

Figure 4. Evolution of average final score for each apparatus (Sierra-Palmeiro et al., 2019)

Very few studies analysing the competition routines in a single major sport event took into account changes in the Code of Points during multiple Olympic cycles (Ávila-Carvalho et al., 
2012; Agopyan, 2014; Bucar et al., 2013). Tsopani et al. (2012) show that the impact of apparatus skills on the final score has increased, but also the coordination between body and apparatus movements, which confers the Code of Points more precision in the performance evaluation criteria. Leandro et al. (2015) and Moskovljević at al. (2013) highlight the increased complexity of the gymnast-apparatus interaction in the routines through an increase in the number of elements or the degree of coordination difficulty.

Referring to the dance steps combination (S), introduced in 2013 as a difficulty element, Reis-Furtado et al. (2020) have pointed out that inserting dance steps in the rhythmic gymnastics routines improves the scores due to body movement, expression and rhythm, which are related to the artistic component. Moreover, the use of dance steps and ballet movements is a consequence of the powerful training models existing in Russia and Eastern Europe, which focus on exquisite technical skills and impeccable postural alignment of the body (Róbeva \& Rankélova, 1991; Vieira De Jesus, 2013).

Batista et al. (2017) analyse the difficulty elements at the 2013 and 2014 Rhythmic Gymnastics World Cup and cluster the routines according to the ranking position in order to emphasise the diversity of difficulty elements and compare these aspects across different value groups, drawing some interesting conclusions: strong similarities between the difficulty elements in top gymnastics routines, which could jeopardise the originality of the composition; the high final score also increased the departure score for difficulty; a large number of mastery elements and additional criteria in the composition of dynamic elements with rotation $(\mathrm{R})$ and throw.

Interesting studies (Leandro, 2018; Leandro et al., 2017) focused on comparing the technical value of the apparatus difficulty (AD) performed in two World Championships, at the beginning of the last two Olympic cycles - 2013 and 2017. The main findings of these studies were: between the World Championships of 2013 and 2017, the body and apparatus difficulty components were more balanced due to the increase in the $\mathrm{AD}$ elements; the $\mathrm{AD}$ distribution in the analysed sport events was as follows: in $2017-54 \% \mathrm{R}$ and $46 \% \mathrm{AD}$; in $2013-78 \% \mathrm{R}$ and 22\% Mastery.

\section{Topic Addressed}

The proposal of the International Federation for the next Olympic cycle is to create a balance between the specific components, namely body difficulty, apparatus difficulty and artistry.

In this context, it is interesting for coaches to point out how the Difficulty criterion can increase the value of exercises and implicitly sport performance; therefore, starting from the identification of decisive factors for top performance in rhythmic gymnastics, as well as the ways to develop and maximise them, the specialised national bodies need to analyse in detail the provisions of the Code of Points and establish pragmatic strategies to exploit the potential of gymnasts within the reference framework mentioned above.

This topical issue highlights the interest of technical experts, coaches and athletes involved in rhythmic gymnastics in adapting training methodologies to changes in the Code of Points.

The current study is addressed to the female junior category, given that, in 2017, the Romanian Rhythmic Gymnastics Federation, in partnership with the Romanian Olympic and 
Sports Committee, established the National Olympic Training Centre for Female Juniors. This structure aimed to harmonise the concept of training with the rigours imposed by the Code of Points in order to increase the chances of athletes to perform at a good level in the targeted competitions. This project was intended to align Romania with the trends of technical regulations issued by the specialised international bodies; therefore, to ensure both the good development of the activity and the achievement of the proposed objectives, it was be necessary to create a specific training strategy for the new regulatory requirements.

The present research is part of an extensive study that highlights an original conceptual and methodological approach by revealing some objective aspects related to all the elements of difficulty and all apparatus for the junior athletes included in the national team.

In the attempt to make a thematic review of the literature by accessing the existing sources in international languages, we noticed a lack of information regarding the detailed analysis of the Difficulty criterion and the pragmatic ways to increase its weight in the final score. From this perspective, further studies on this topic are needed, which engage, in a collaborative effort, specialists from different countries or different national federations.

\section{Development trends in elite rhythmic gymnastics}

The development trends in elite rhythmic gymnastics are determined by changes in the FIG Code of Points. Basically, changes occur as a result of analysing major competitions in order to assess both quantitatively and qualitatively the level of training of gymnasts, the impact on the public and the efficiency of the evaluation system.

The FIG Technical Committee aims to standardise the training of gymnasts and set clear objectives that help meet the performance criteria required by the Code of Points.

The CoP is intended for two levels of training, namely junior and senior, and is valid for official international competitions. In each country, the national federation has the right to design its own internal technical regulations, but the requirements usually remain the same for the junior and senior categories, while for the children categories, the requirements are designed according to their level of training.

A problem reported in rhythmic gymnastics over the past few years is the duration of the rules governing this sport. Despite the fact that the regulation must be amended every 4 years at the end of an Olympic cycle, it undergoes annual or even semestrial changes that are presented to national federations, coaches and judges through letters issued by the International Gymnastics Federation. This has consequences on the consistency with which coaches design longer-term training and disrupts the psycho-emotional balance of gymnasts.

The latest edition of the CoP is valid for the 2017-2020 Olympic cycle. It presents the current level of development of elite rhythmic gymnastics, expressing the requirements and new trends in this sport.

The variables that make up the CoP represent the specific evaluation criteria, namely the Difficulty criterion and the Execution criterion, which in turn are subdivided. Throughout the years, the structure of evaluation criteria has been changed in order to emphasise the importance of each one and improve the evaluation of gymnasts by judges. 
The Difficulty criterion includes all the techniques of body movements and apparatus handling elements (apparatus technical groups) that make up a competition routine. The level of this criterion measures the value of gymnasts (Leandro et al., 2016).

In order to establish the difficulty elements that make up a routine, coaches need to consider the level of training of athletes, because any discrepancy between the two variables can lead to major errors. These elements must be performed with accuracy and a high aesthetic level (Hashimoto et al., 2017).

The Execution criterion refers to the correctness and the way in which a gymnast performs the competition routine. It takes into account both the technical and artistic aspects, which are considered to be two distinct evaluation sub-criteria.

The correctness with which a gymnast technically performs both the body elements and apparatus handling elements is evaluated by the Technical Execution sub-criterion. Any deviation will be considered a technical fault and will be penalised by deduction of points.

The artistic component is the one that maintains this sport at the border with art through the theme of the exercise and the means of expression, which are able to display the compositional idea. It must create a unitary artistic representation from the beginning to the end, which is expressed through body movements performed in a fluid way, without moments of discontinuity. Any deviation from these principles will be penalised depending on the severity of composition or execution errors by applying deductions for artistic faults.

Regulatory requirements imposed by the FIG Code of Points in the periods 2005-2008 and 2009-2012

Between 2001 (after the Sydney Olympics) and 2008, it was found that the trends in the Code of Points were focused on developing the technique of body movements. In order to meet regulatory requirements, athletes had to perform a large number of elements during an exercise lasting 1:30 minutes, which resulted in excessive stress on gymnasts, with negative repercussions on their health status.

The combination of elements performed successively, without interruption, jeopardised the artistic image of the routines, pushing rhythmic gymnastics away from its origins because the synchronisation between music and movement became difficult (Manos, 2008).

The artistic component was evaluated together with the technical elements of apparatus handling, 9 points being awarded for choreographic aspects, which also included mastery and originality elements, and 1 point for aspects related to musical accompaniment, to creating a symbiosis between music, body movement and apparatus handling (Chiat \& Ying, 2012).

Of the 9 points, 2 were awarded for "compositional aspects", namely identifying the basic idea, creating a balance in apparatus handling with the right hand and the left hand, ensuring variety in trajectories, directions of movement and levels, using the competition area and choosing the technical elements of apparatus handling, the technical elements of body difficulty and the pre-acrobatic elements. Another 7 points were awarded for the apparatus handling elements. Mastery elements could be awarded when identifying a base for apparatus handling and a certain criterion. For example, when bouncing the ball with one part of the lower limb, the bounce is the base, and the criterion is granted due to the fact that it is performed with another part of the body than the hand. 
The formula for calculating the total score is (2005-2008 CoP, FIG, 2007):

$\mathrm{E}+((\mathrm{A}+\mathrm{D}) / 2)=$ Final score $($ a maximum of 20 points $)$

where: E represents the execution score, which can reach a maximum of 10 points, $A$ is the score for the artistic component (a maximum of 10 points) and D is the difficulty score (body difficulty + apparatus difficulty), which can reach a maximum of 10 points.

After analysing the Beijing Summer Olympics, the Rhythmic Gymnastics Technical Committee decided again to reduce the number of body difficulty elements and apparatus difficulty elements from a maximum of 18 to a maximum of 12 , which, added together, could reach 10 points.

Since 2009, the constant trend has been to reduce the number of body difficulty elements, update the types of difficulties and invigorate the aspects related to the apparatus technique and the Artistic criterion (Ávila-Carvalho et al., 2012; Trifunov \& Dobrijevic, 2013).

For these two editions of the Code of Points, the fundamental groups of body movement elements (valid for difficulties) were focused on: jumps/leaps, balances, pivots, flexibilities/ waves, each one being specific to an apparatus within the competition programme as follows:

- $\quad$ rope - jumps and pivots;

- hoop - all body movement groups are compulsory;

- ball - flexibilities/waves and jumps;

- clubs - balances and pivots;

- ribbon - pivots and jumps.

Starting 2009, the Code of Points has been improved firstly by reducing the number of body difficulty elements and secondly by restructuring the evaluation system.

The difficulty is evaluated independently according to two distinct sub-criteria: one concerns all elements related to the technique of body movements (D1), and the other refers to all elements belonging to the apparatus technique (D2).

The artistic component will only consider the creative act, taking into account the following aspects:

- musical accompaniment;

- $\quad$ artistic image (the idea of the choreographic act);

- expressivity (displaying and transmitting the choreographic idea).

The formula for calculating the total score is (2009-2012 CoP, FIG, 2009):

$(\mathrm{D} 1+\mathrm{D} 2) / 2+\mathrm{A}+\mathrm{E}=$ Final score (a maximum of 30.00 points)

where: D1 represents the total value related to the technique of body difficulty elements, D2 is the total value related to the apparatus technical difficulty elements, each sub-category being able to gather a maximum of 10 points. A represents the artistic component, which can reach the value of 10 points, and $\mathrm{E}$ refers to the technical execution.

\section{Regulatory requirements imposed by the 2013-2016 and 2017-2020 FIG Codes of Points}

The 2013 Code of Points brought the biggest changes from a regulatory point of view, receiving positive feedback from specialist technicians, with little controversy regarding the elimination of the fundamental body group "flexibilities and waves" or the judging sheet that 
chronologically recorded all the elements of difficulty within the composition of a competition routine (Agopyan \& Serdil Ors, 2019).

The penultimate edition of the Code of Points brought substantial changes in terms of both quantifying the structure of the difficulty evaluation criterion and increasing the appreciation of aspects related to the artistic component.

If previous regulations had been focused on increasing the number of body difficulty elements and improving them, in the 2013-2016 Code of Points (FIG, 2013), the number of body difficulty elements decreased, new types of apparatus technical elements (called apparatus mastery elements) were introduced and a detailed definition of the Artistic subcriterion was attempted. All these updates implemented by the FIG Technical Committee were aimed at bringing rhythmic gymnastics back to its origins and attracting the public through spectacular competition routines.

From a desire to simplify the judging system and reduce the number of difficulty elements and therefore the overuse of gymnasts, it was decided that the total score for the Difficulty criterion should be reduced to 10 points, including both body difficulty elements and apparatus difficulty elements.

Body difficulty elements underwent the most important changes: the maximum permissible number of elements further decreased to 9, with a minimum of 6 compulsory elements; the variety of body difficulty elements also decreased, and the fundamental body group "flexibilities and waves" was eliminated, most of the elements being distributed in the rotation and balance groups.

The dance steps combination, also present in the previous years, received a clear definition, specific features and a value under the Difficulty criterion. It had to be performed within 8 seconds in accordance with the music and compositional idea, in a fluid way, without interruption, in coordination with at least one fundamental technical element specific to each apparatus and without technical execution faults. Assigning a quantified value was aimed at increasing the artistic level of the compositions.

Apparatus technical elements, as part of the Difficulty evaluation criterion, included only dynamic elements with rotation and throw and apparatus mastery elements.

Dynamic elements with rotation and throw consist of a large throw of the apparatus plus minimum 2 rotations of the body, which can be performed during the throw, under and during the catch of the apparatus. In order to capitalise on the total value of these elements, other criteria can be added, which will increase the difficulty of the exercise.

Apparatus mastery elements represent the great novelty of this Code of Points and refer to the execution of unusual combinations of apparatus handling elements, which are valid only when performed without any technical execution fault. These combinations of elements must include minimum one base (fundamental and/or non-fundamental apparatus technical element) and two criteria or two bases and one criterion, having a standard value of 0.20 points.

From a desire to motivate coaches and gymnasts and increase the spectacularity of the routines and attract the public, the execution of a single exercise with musical and vocal accompaniment was allowed.

After the Rio Olympics, the Technical Committee conducted a new analysis in order to improve the regulation and evaluation system. 
Thus, the current regulation that revolutionises elite rhythmic gymnastics appears, namely the 2017-2020 FIG Code of Points, which has substantially improved its latest version.

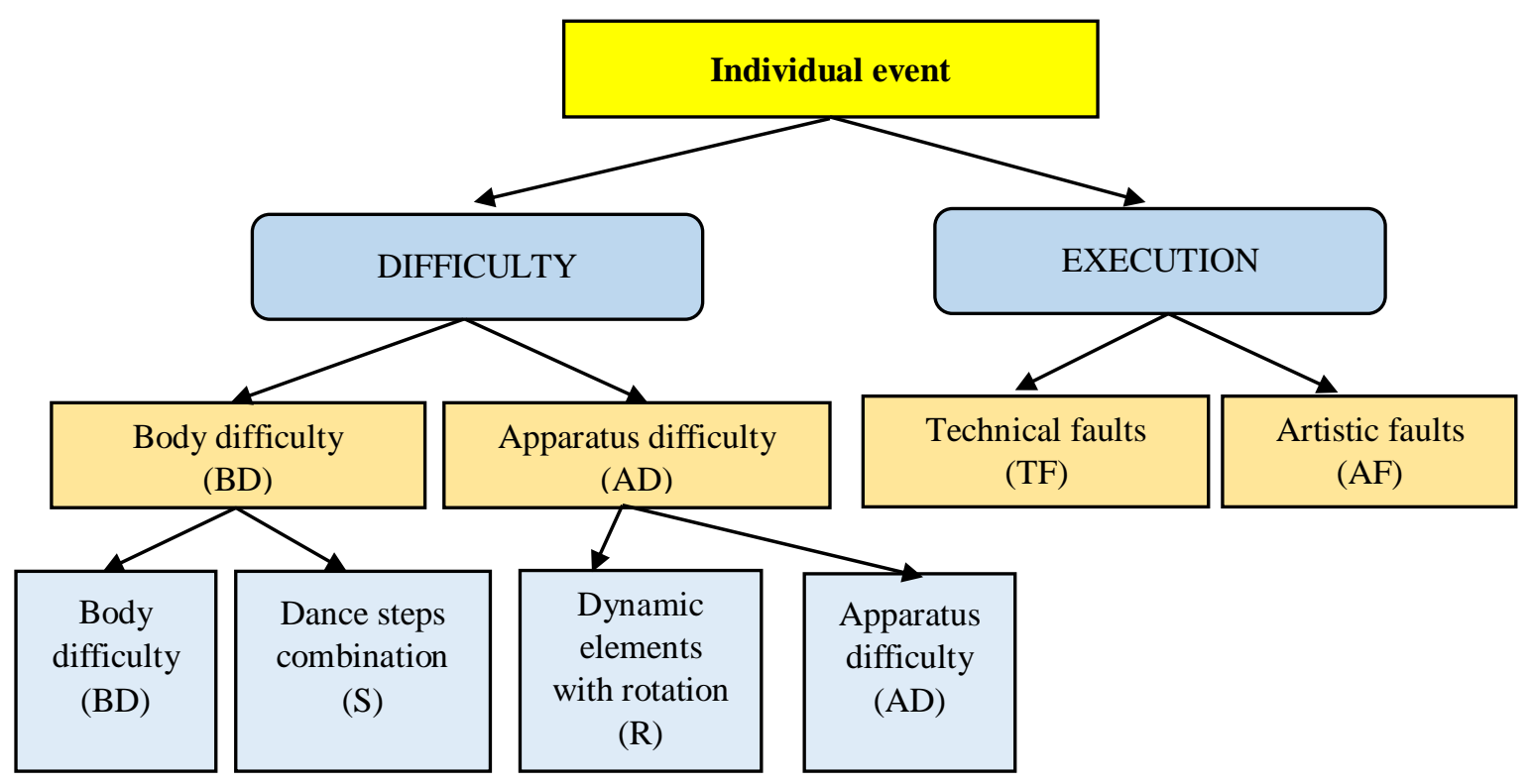

Figure 5. Evaluation criteria specific to the individual event in the current FIG Code of Points (2017-2020)

The most important aspect in terms of evaluation is the elimination of the judging sheet, which included all the technical difficulty elements of an exercise, the gymnast having the possibility to perform only the elements written on that document before the start of the competition, any other element that was performed but undeclared being ignored by judges.

New definitions of apparatus mastery also appear, its name changing to apparatus difficulty elements. They are defined as technically difficult apparatus-body synchronisation or an interesting or innovative use of the apparatus, which are differently handled compared to the basic technique specific to rhythmic gymnastics; they can have values between 0.20 and 0.40 points, depending on the criteria and their difficulty.

At the end of 2017, after the first edition of the World Championships in this Olympic cycle, some monotony and standardisation of the exercises were noticed due to the repetition of body difficulty elements and apparatus handling, but also to the limitation of the technical accuracy and the virtuosity specific to rhythmic gymnastics. In these conditions, it was decided to eliminate the 10-point limit from the Difficulty criterion, redefine apparatus difficulty elements and substantially increase the deduction values for technical faults and artistic faults.

Particular attention is paid to the artistic component, which is independently evaluated by two judges who must take into account the following regulatory requirements:

a) Unity of composition - it refers to the development of a coherent artistic image or idea that has to be found from the beginning to the end of the exercise and is expressed through body and apparatus movements.

- Guiding idea (character) - it must be supported by creating a movement style specific to the musical accompaniment. If there is any change in the character of the music, it 
must also be found in the character of the gymnast's movements. It is recommended to introduce the compositional idea in the first part of the exercise, to change the rhythm/dynamism in the middle part (where the composition develops) and, in the final part, the music must increase in intensity, the exercise ending with a distinct pose.

- Connections - they create continuity between the technical elements so that there are no moments of pause or abrupt transitions from one element to another. In this way, the use of different levels and directions of body movements and apparatus planes must highlight the fluidity of the composition.

b) Music and movement must be in continuous connection:

- body and apparatus movements must be correlated with the musical accents and phrases, by explicitly emphasising the rhythm of the music.

- dynamic changes in the intensity and speed of movements will be performed in accordance with the musical accompaniment. If the music does not have this feature, the gymnast can create her own change of dynamism, which is highlighted by the ways of motor expression.

c) Body expression - it must exist throughout the exercise, including facial expressions, the gymnast performing the elements, connections and dance steps with maximum amplitude and plasticity.

d) Variety in directions, trajectories, planes, levels, modalities of traveling and apparatus elements - they will contribute to avoiding the monotony of the exercise.

In order to decide between gymnasts of very close values, penalties for technical deductions have been increased. Consequently, there was a substantial decrease in the scores obtained at the beginning of 2018 compared to those obtained in 2017. Technical faults for body difficulty elements are divided into three categories: small, medium and large, depending on the deviation from the basic technique of the element. Based on the same principle, we can also see an increase in penalties for apparatus faults, in case of failure or deviation from the correct trajectory.

Thus, technicians have to attach great importance to correcting technical execution faults, helping gymnasts to properly learn the technique of performing body movements and apparatus handling elements, as well as increasing their execution speed in order to improve their final scores for the Difficulty criterion.

\section{Conclusion}

The 2017-2020 FIG Code of Points provides, on the one hand, the elimination of the 10point limit for the Difficulty criterion and, on the other hand, the redefinition of apparatus difficulty elements from the perspective of apparatus-body synchronisation and innovative use of the apparatus, without numerical or structural limitations of the movement.

The development trends in elite rhythmic gymnastics are determined by changes in the Code of Points issued by the International Gymnastics Federation, which, after major competitions, assesses both quantitatively and qualitatively the level of training of gymnasts, as well as the impact on the public, and makes changes to the evaluation criteria with a view 
to increase the spectacularity of the exercise, in the sense of redefining the apparatus difficulty elements and creating an unlimited space of creativity for them, both numerically and as a movement structure.

In order to build a strategy to improve the performance of Romanian gymnasts in international competitions, a careful analysis of the exercises performed in large-scale competitions is required. The data extracted and interpreted can represent an important resource of information through which technicians can analyse the new trends in this sport and adapt the provisions of the FIG Code of Points so that the potential of each gymnast can be maximally exploited.

\section{References}

Agopyan, A. (2014). Analysis of body movement difficulties of individual elite rhythmic gymnasts at London 2012 Olympic Games Finals. Journal of Scientific Research, 19(12), 1554-1565. http://doi.org/10.5829/idosi.mejsr.2014.19.12.81263

Agopyan, A., \& Serdil Ors, B. (2019). An analysis of variations in body movement difficulty of 2016 Olympic Games rhythmic gymnast candidates. International Journal of Performance Analysis in Sport, 19(9), 417-434. https://doi.org/10.1080/24748668.2019.1617017

Ávila-Carvalho, L., Klentrou, P., Palomero, M., \& Levre, E. (2012). Analysis of the technical content of elite rhythmic gymnastics group routines. Open Sports Science Journal, 5, 146153. http://dx.doi.org/10.2174/1875399X01205010146

Batista, A., Garganta, R., \& Ávila-Carvalho, L. (2017). Dance steps, dynamic elements with rotation and throw and mastery elements in rhythmic gymnastics routines. Science of Gymnastics Journal, 9(2), 177-189.

Bucar, M., Cuk, I., Pajek, J., Kovac, M., \& Leskosek, B. (2013). Is the quality of judging in women artistic gymnastics equivalent at major competitions of different levels? Journal of Human Kinetics, 37, 173-181. https://dx.doi.org/10.2478\%2Fhukin-2013-0038

Chiat, L. F., \& Ying, L. F. (2012). Importance of music learning and musicality in rhythmic gymnastics. Procedia - Social and Behavioral Sciences, 46, 3202-3208. https://doi.org/10.1016/j.sbspro.2012.06.037

FIG. (2007). 2005-2008 Code of Points. Rhythmic gymnastics. https://www.japan-rg.com/lineup/event/page/competiton/child12/item/coderg0701-e2.pdf

FIG. (2009). 2009-2012 Code of Points. Rhythmic gymnastics. https://www.yumpu.com/en/document/view/49515769/code-of-points-rhythmicgymnastics-2009-aaeura-2012

FIG. (2013). 2013-2016 Code of Points. Rhythmic gymnastics. https://www.scottishgymnastics.org/sites/default/files/imce/disciplines/2012\%20$\% 202016 \% 20$ Rhythmic\%20Code\%20of\%20Points.pdf

FIG. (2018). 2017-2020 Code of Points. Rhythmic gymnastics (with Errata dec. 2017, valid through 31 dec. 2021). https://www.gymnastics.sport/publicdir/rules/files/en_RG\%20CoP\%2020172020\%20with\%20Errata\%20Dec.\%2017.pdf

Hashimoto, M., Kida, N., \& Nomura, T. (2017). Characteristics of women's rhythmic gymnastics from the perspective of "body difficulty" and performance time. Advances in Physical Education, 7(3), 260-273. https://doi.org/10.4236/ape.2017.73021

Leandro, C. (2018). Apparatus difficulty in rhythmic gymnastics routines - Comparison between 2 Olympic cycles. Science of Gymnastics Journal, 10(3), 413-419. 
Leandro, C., Ávila-Carvalho, L., Sierra-Palmeiro, E., \& Bobo-Arce, M. (2015). Accuracy in judgment the difficulty score in elite rhythmic gymnastics individual routines. Science of Gymnastics Journal, 7(3), 81-93.

Leandro, C., Ávila-Carvalho, L., Sierra-Palmeiro, E., \& Bobo-Arce, M. (2016). Departure difficulty score vs final difficulty score. The effect of performance in elite rhythmic gymnastics. Athens Journal of Sports, 3(3), 169-178. https://doi.org/10.30958/ajspo.3-3-1

Leandro, C., Ávila-Carvalho, L., Sierra-Palmeiro, E., \& Bobo-Arce, M. (2017). Judging in rhythmic gymnastics at different levels of performance. Journal of Human Kinetics, 60, 159-165. https://dx.doi.org/10.1515\%2Fhukin-2017-0099

Manos, M. (2008). Gimnastica ritmică de performanță [Performance rhythmic gymnastics]. Bren.

Moskovljević, L., Radisavljević, L., \& Dabović, M. (2013). The speed of progress in the apparatus handling technique in rhythmic gymnastics. Fizička Kultura, 67(1), 33-39. https://doi.org/10.5937/fizkul1301033M

Reis-Furtado, L. N., Toledo, E., Fernandes-Antulpa, K., \& Carbinatto, M. V. (2020). Ballet movements in rhythmic gymnastics routines: An analysis from the last two Codes of points (2013-2016 and 2017-2020). Science of Gymnastics Journal, 12(3), 395-406.

Róbeva, N., \& Rankélova, M. (1991). Escola de campeãs: Ginástica rítmica desportiva [The school of champions: Sport rhythmic gymnastics] (4th ed.). Ícone.

Sierra-Palmeiro, E., Bobo-Arce, M., Perez-Ferreiros A., \& Fernandez-Villarino, M. (2019). Longitudinal study of individual exercise in elite rhythmic gymnastics. Frontiers in Psychology, 10: 1496. https://doi.org/10.3389/fpsyg.2019.01496

Trifunov, T., \& Dobrijevic, S. (2013). The structure of difficulties in the routines of the best world and Serbian rhythmic gymnasts. Physical Culture, 67(2), 120-129. https://doi.org/10.5937/fizkul1302120T

Tsopani, D., Dallas, G., Tasika, N., \& Tinto, A. (2012). The effect of different teaching system in learning rhythmic gymnastics apparatus motor skills. Science of Gymnastics Journal, 4, 55-62.

Vieira de Jesus, D. S. (2013). Réquiem para dois pássaros de gelo: A coreografia da exclusão na patinação artística soviética e a construção da potência esportiva mundial [Requiem for two ice birds: The choreography of exclusion in Soviet figure skating and the construction of the world's sports power]. Revista Estudos Feministas, 21(3), 1211-1230.

https://doi.org/10.1590/S0104-026X2013000300024 